\title{
Correction and addendum to "The formation of clusters during large sputtering events" [Nucl. Instr. and Meth. B 124 (1997) 31]
} John W. Hartman, Mark H. Shapiro ${ }^{1}$, T.A. Tombrello *

Department of Physics, Mathematics, and Astronomy 200-36, California Institute of Technology, Pasadena, CA 91125, USA

Received 20 June 1997

We write to note a correction to the event-sizespecific uncorrelated recombination model presented in "The formation of clusters during large sputtering events" by Hartman et al. [1]. In that paper, Eq. (4), the expression for the phase space density of recombined $k$-clusters, is in error. Correction affects the relative normalization of $k$ cluster spectra, but not their functional forms. Expressions requiring correction include: the total yield of $k$-clusters in an event of size $N$, the absolute yield of $k$-clusters, and the normalization of the energy and angular spectra. Not affected by the correction are the functional forms of the energy and angular spectra or the predicted dependence of small $k$-cluster production on event size $N: Y_{k}(N) \propto N^{k}$.

The primary assumption of the uncorrelated recombination model (as presented in Ref. [1]) is as follows: the probability that any 1 of the $N$ atoms ejected during a single sputtering event leaves from a given site, $\boldsymbol{x}$, with a given momentum, $\boldsymbol{p}$, at a

\footnotetext{
* Corresponding author. Tel.: 626395 4581; fax: 626683 9060; e-mail: tat@cco.caltech.edu.

${ }^{1}$ Permanent address: Department of Physics, California State University Fullerton, CA 92634, USA.
}

given time, $t$, is independent of the ejection sites, momenta, and times of the other $N-1$ sputtered atoms. Assuming that sputtering of the $N$ atoms is described by a phase space density $\mathrm{d} N / \mathrm{d} \gamma=\mathrm{d} N / \mathrm{d}^{3} x \mathrm{~d}^{3} p \mathrm{~d} t$, the probability that any 1 of the $N$ sputtered atoms leaves the target from within a small phase space volume $\Delta \gamma=$ $\Delta^{3} x \Delta^{3} p \Delta t$ about $\gamma=(\boldsymbol{x}, \boldsymbol{p}, t)$ is $(\Delta \gamma / N)(\mathrm{d} N / \mathrm{d} \gamma)$. The recombination assumption posits that if $k$ atoms are sputtered from within a small enough region $\Delta \gamma$, then the interatomic potential will bind them together into a $k$-cluster. Simple combinatorics imply that the probability that $k$ of $N$ atoms are sputtered from $\Delta \gamma$ about $\gamma$ is

$$
\begin{aligned}
\Delta \gamma \frac{\mathrm{d} Y_{k}(N)}{\mathrm{d} \gamma} \simeq & \frac{N !}{(N-k) ! k !} \\
& \times\left(1-\frac{\Delta \gamma}{N} \frac{\mathrm{d} N}{\mathrm{~d} \gamma}\right)^{N-k}\left(\frac{\Delta \gamma}{N} \frac{\mathrm{d} N}{\mathrm{~d} \gamma}\right)^{k},
\end{aligned}
$$

where $\mathrm{d} Y_{k}(N) / \mathrm{d} \gamma$ is the phase space density of recombined $k$-clusters with phase per atom $\gamma=(\boldsymbol{x}, \boldsymbol{p}, t)$. Eq. (4) of Ref. [1] identifies the normalization of this phase space density to be per cluster and is incorrect. Potential (and actual) confusion about the normalization of $\mathrm{d} Y_{k}(N) / \mathrm{d} \gamma$ is settled by noting that the sum rule, 
$\frac{\mathrm{d} N}{\mathrm{~d} \gamma}=\sum k \frac{\mathrm{d} Y_{k}(N)}{\mathrm{d} \gamma}$

must be satisfied. In this simple model, this sum rule restricts the recombination volume $\Delta \gamma$ to be independent of cluster size.

The recombination volume is (as in Ref. [1]) determined by the binding distance, $a$, and depth, $-V$, of the interatomic potential, and the time a cluster requires to travel the binding distance: $\Delta^{3} x \sim a^{3}, \Delta^{3} p \sim(2 m V)^{3 / 2}, \Delta t \sim m a / p$, so $\Delta \gamma=$ $z a^{3}(2 m V)^{3 / 2} m a / p$, where $m$ is the mass of an atom in the cluster, $p$ its momentum, and $z$ a free parameter of order 1 introduced by the crudeness of this approximation. The same assumptions about the separability of $\mathrm{d} N / \mathrm{d}_{\gamma}$ and the separated densities as in Ref. [1] are made. Namely: the spatial volume from which individual atoms are sputtered is $\Omega$; their momenta are described by a modified Sigmund-Thompson distribution [2]; and their rate of sputtering slows exponentially after the initial impact. Corrected expressions for the distribution and absolute expectation of $k$-clusters from an event of size $N$ are:

$$
\begin{aligned}
\frac{\mathrm{d} Y_{k}(N)}{\mathrm{d} \epsilon \mathrm{d}(\cos \theta)} \simeq & \frac{1}{k} \frac{N !}{(N-k) ! k !} n(n-1) \\
& (q+1)(z \alpha)^{k-1} \frac{\epsilon}{U^{2}}\left(\frac{\cos ^{q} \theta}{(1+\epsilon / U)^{n+1}}\right)^{k} .
\end{aligned}
$$

$$
\begin{aligned}
Y_{k}(N) \simeq & \frac{N !}{(N-k) ! k !} \frac{(z \alpha)^{k-1}}{k} \\
& \frac{n(n-1)(q+1)}{[k(n+1)-1][k(n+1)-2](k q+1)} .
\end{aligned}
$$

Here $\epsilon$ is the kinetic energy per atom of the $k$-cluster, related to the cluster kinetic energy by $E=k \epsilon$,

$\alpha=\frac{n(n-1)(q+1)}{\pi} \frac{a^{3}}{\Omega}\left(\frac{V}{U}\right)^{3 / 2} \frac{m a / \tau}{\sqrt{2 m U}}$

is a dimensionless constant set by the ratio of recombination to total-sputtering phase space volumes, and the assumption $(1-(\Delta \gamma / N)$ $(\mathrm{d} N / \mathrm{d} \gamma))^{N-k} \simeq 1$ has been made.

In Ref. [1] the distribution of event sizes was observed to decay exponentially with size: $P(N) \simeq$ $\mathrm{e}^{-\beta N}\left(1-\mathrm{e}^{-\beta}\right)$ with $\beta \simeq 0.22$. Hence, during a sputtering event of indeterminate size, the expected distributions and absolute yields of $k$-clusters are:

$$
\begin{aligned}
\frac{\mathrm{d} Y_{k}}{\mathrm{~d} E \mathrm{~d}(\cos \theta)}= & \sum_{N} P(N) \frac{1}{k} \frac{\mathrm{d} Y_{k}(N)}{\mathrm{d} \epsilon \mathrm{d}(\cos \theta)} \\
= & \frac{1}{k} \frac{1}{\left(\mathrm{e}^{\beta}-1\right)^{k}} \frac{(z \alpha)^{k-1} n(n-1)(k q+1)}{k} \\
& \times \frac{\epsilon}{U^{2}}\left(\frac{\cos ^{q} \theta}{(1+\epsilon / U)^{n+1}}\right)^{k}
\end{aligned}
$$

$$
\begin{aligned}
\frac{\mathrm{d} Y_{k}}{\mathrm{~d} E}= & \frac{1}{k} \frac{1}{\left(\mathrm{e}^{\beta}-1\right)^{k}} \frac{(z \alpha)^{k-1} n(n-1)(q+1)}{k(q k+1)} \\
& \times \frac{\epsilon}{U^{2}} \frac{1}{(1+\epsilon / U)^{k(n+1)}},
\end{aligned}
$$

$$
\begin{aligned}
\frac{\mathrm{d} Y_{k}}{\mathrm{~d}(\cos \theta)}= & \frac{1}{\left(\mathrm{e}^{\beta}-1\right)^{k}} \frac{(z \alpha)^{k-1}}{k} \\
& \times \frac{n(n-1)(q+1)}{[k(n+1)-1][k(n+1)-2]} \cos ^{k q} \theta,
\end{aligned}
$$

$$
\begin{aligned}
Y_{k}= & \frac{1}{\left(\mathrm{e}^{\beta}-1\right)^{k}} \frac{(z \alpha)^{k-1}}{k} \\
& \times \frac{n(n-1)(q+1)}{[k(n+1)-1][k(n+1)-2][k q+1]} .
\end{aligned}
$$

Note that the functional forms of the differential spectra are the same as those predicted by other recombination models [3] but prediction of the normalizations requires knowledge of the distribution of event sizes and the effectiveness of the interatomic potential when recombining the sputtered atoms into clusters.

In Ref. [1], simulations of $3 \mathrm{keV} \mathrm{Ar} \rightarrow$ In-Ga (liquid) sputtering events were simulated and the emission statistics collected. The exponents $n$ and $q$, and the 'binding energy' $U$ are determined by fitting the monomer spectra to the generalized Sigmund-Thompson form, $\alpha$ is determined by these parameters and the interatomic potential, and $\beta$ is determined by the distribution of event sizes (see Ref. [1]). The only free parameter in the model is $z$, which has been adjusted to match the relative yields of dimers and monomers to that observed 
during the simulations. In Fig. 1, $Y_{k}$ observed during the simulations and predicted by the uncorrelated recombination model are compared; this figure replaces Fig. 8 of Ref. [1]. The recombination predictions are seen to fit the observed relative yields of $k$-clusters and to follow a power law decay for clusters of size $k \simeq 6$ and smaller; in Ref. [1] the yield was thought to decay must faster. In Fig. 2 the differential spectra for monomers dimers and trimers observed during the simulation and predicted by the uncorrelated recombination model are compared; this figure replaces Fig. 9 of Ref. [1].

The correction of our expression for the phase space density of recombined $k$-clusters and the subsequent development of predicted yields and spectra is significant because it suggests that the yield of small $k$-clusters from uncorrelated recombination processes is greater than our previous (incorrect) results indicated. Before, even for the small cluster size $k=4$, one could discern a divergence of the model's predictions of the absolute yield from that which we found. With the corrected model, we find that we can fit adequately the absolute yield of small $k$-clusters without re-

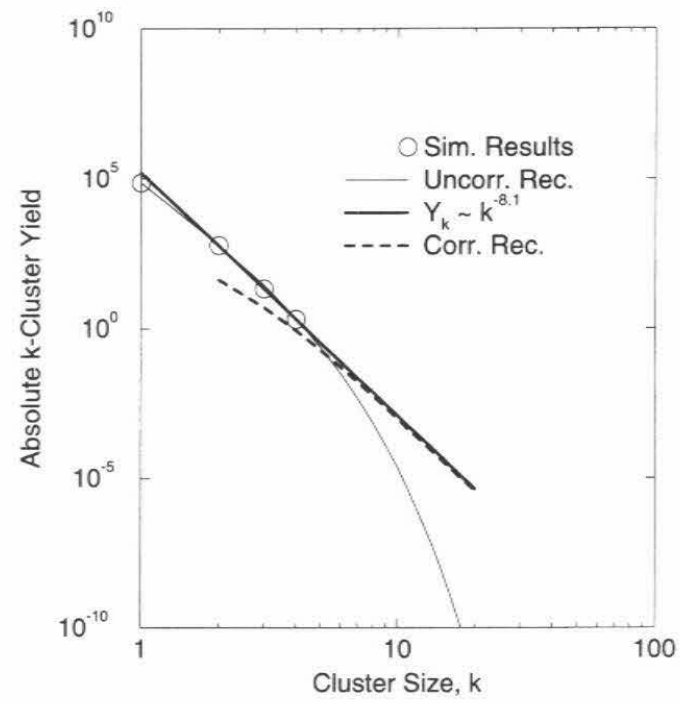

Fig. 1. The yield of $k$-clusters in the simulation, the corrected recombination prediction, and the power law fit $Y_{k} \sim k^{-8.1}$. Note that uncorrelated recombination is as robust as the power law for $k<6$.

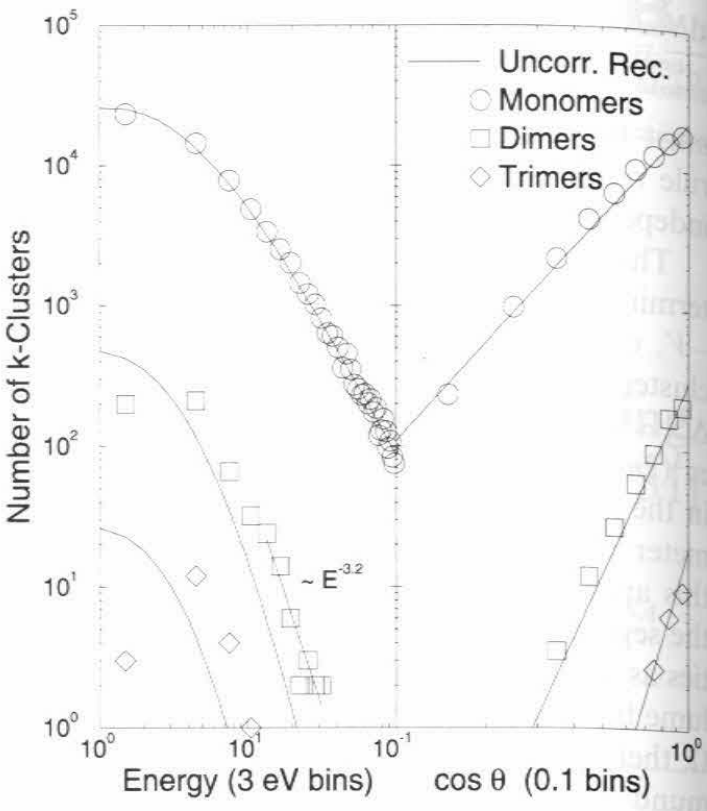

Fig. 2. (a) The energy spectra of clusters and the corrected recombination prediction. The yield of high energy clusters is still underestimated by the uncorrelated recombination model. (b) The angular spectra of clusters and the recombination prediction.

quiring a significant contribution from correlated processes. Indeed, if one assumes that a power law decay of $Y_{k}$ with $k$ is valid, then the uncorrelated recombination model seems adequate up until $k=6$ (see Fig. 1). The predicted energy spectra of the $k$-clusters are still unsatisfactory. Uncorrelated recombination does not predict enough high energy $k$-clusters, and overestimates the number of low energy $k$-clusters (see Fig. 2).

\section{References}

[1] J.W. Hartman, M.H. Shapiro, T.A. Tombrello, Nucl. Instr. and Meth. B 124 (1997) 31.

[2] P. Sigmund, Phys. Rev. 184 (1969) 383; M.W. Thompson, Phys. Rep. (Rev. Sec. of Phys. Lett.) 69 (4) (1981) 335.

[3] W. Gerhard, Z. Phys. B 22 (1975) 31; G.P. Konnen, A. Tip, A.E. deVries, Radiat. Eff. 21 (1974) 269; G.P. Konnen, A. Tip, A.E. de Vries, Radiat. Eff. 26 (1975) 23. 